\title{
Microfiber loop resonator based temperature sensor
}

\section{S. W. Harun \\ swharun@um.edu.my}

\author{
University of Malaya, Department of Physics, Photonics Research Center, 50603 Kuala Lumpur, \\ Malaysia \\ University of Malaya, Department of Electrical Engineering, 50603 Kuala Lumpur, Malaysia
}

University of Malaya, Department of Electrical Engineering, 50603 Kuala Lumpur, Malaysia

University of Malaya, Department of Physics, Photonics Research Center, 50603 Kuala Lumpur, Malaysia
K. S. Lim

\section{S. S. A. Damanhuri}

\section{H. Ahmad}

A microfiber loop resonator (MLR) is fabricated by coiling a microfiber which is fabricated using a flame heating technique, into itself. A temperature response on a comb spectrum of a fabricated MLR, which is embedded in a low refractive index polymer, is investigated. The spacing of the transmission comb spectrum of the MLR is observed to be unchanged with the temperature. However, the extinction ratio of the spectrum is observed to be linearly decreased with the temperature. The slope of the extinction ratio reduction against temperature was about $0.043 \mathrm{~dB} /{ }^{\circ} \mathrm{C}$. The dependence of the extinction ratio on temperature is due to the change in the material's refractive index. [DOI: 10.2971/jeos.2011.11026]

Keywords: microfiber loop resonator, temperature sensor, microfiber devices

\section{INTRODUCTION}

Optical micro-ring resonators have attracted considerable attention owing to their simple structure, compact size, and many applications in communication, optical signal processing and optical devices [1]-[2]. Planar waveguide microring resonators have been well developed, but suffer from larger connection-losses with fibers and higher cost. Recently, research on low-loss microfibers has opened up new opportunities for developing micro-photonic devices such as resonators [3], couplers [4], and sensors [5]. As one of the basic functional elements, microfiber resonators, in forms of loops, knots, or coils, have shown high intrinsic optical quality and have exhibited promising applications such as filters and lasers [6].

Fiber-optic sensors have found increasing applications in different technological fields. Various types of fiber sensors have been proposed and demonstrated [7]-[9]. Microfiberbased sensors are amongst the simplest of such devices, as they do not require expensive and complex fabrication procedures. In contrast, fiber Bragg grating sensors, for example, require expensive phase-masks. Recently, strain, high temperature and refractive index sensing using tapered microstructured optical fiber have been reported [10]-[12]. However, the authors used tapered micro-structured fiber which is expensive and difficult to work with in term of getting low loss splicing with standard optical fiber.

In this paper, a temperature sensor is demonstrated using a microfiber loop resonator (MLR), which is fabricated using a microfiber with a few micrometers in diameter. The microfiber is fabricated by heating and stretching a piece of standard silica single-mode fiber (SMF), after the polymer protective cladding has been removed. The MLR is formed by coiling the microfiber onto itself and the device is embedded in a low refractive index material for robustness. The proposed temperature sensor device has a low loss splicing with a standard SMF.

\section{EXPERIMENT}

A tapered fiber is fabricated using an experimental set-up shown in Figure 1. A coating removed standard SMF was held by two fiber holders which were fixed on two translation stages. One stage is fixed and another stage is a motorized stage that can be moved in one dimensional direction and the speed of the motor can be controlled. The fiber ends were connected to an amplified spontaneous emission (ASE) source and an optical spectrum analyzer (OSA). The tapered fiber was fabricated by heating the fiber to its softening temperature, and then pulling the ends apart to reduce the fiber's diameter down to a around 1-3 $\mu \mathrm{m}$. A high temperature and stable micro-burner fueled by clean butane gas is used in the tapering. Mounted on the movable stand, the micro-burner can be swung vertically to flame brush the tapered fiber. The flame should be clean and the burning gas 


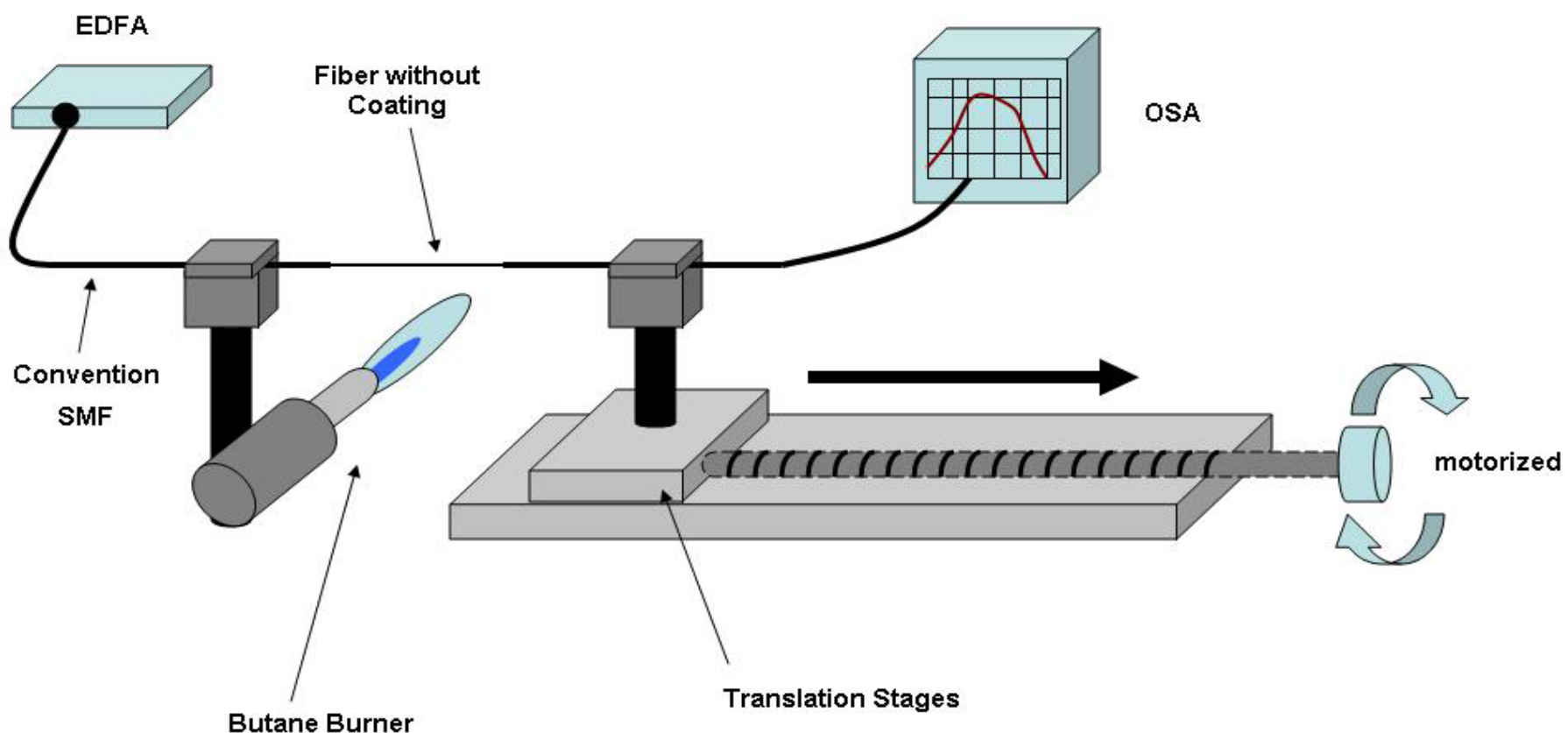

FIG. 1 Schematic diagram of the microfiber fabrication set-up.

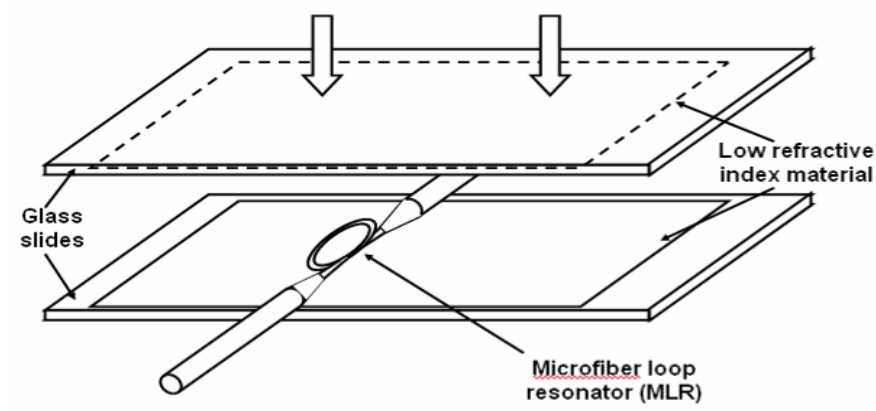

a) diagram

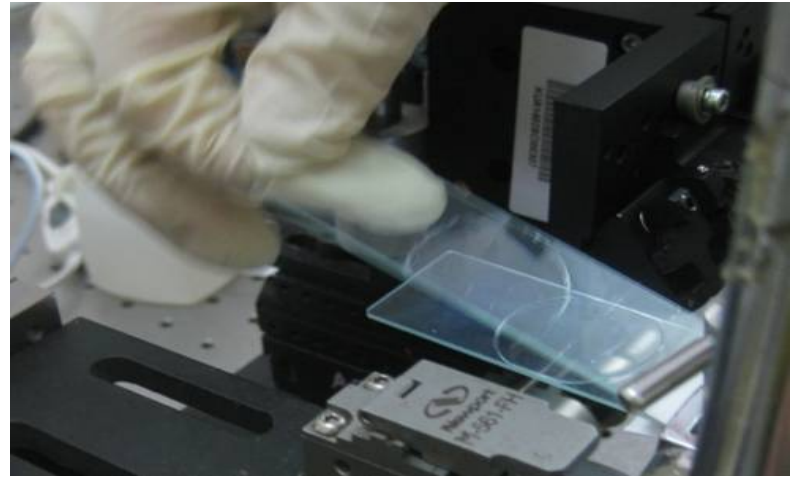

b) snapshot

FIG. 2 Packaging of the MLR a) schematic diagram b) snapshot.

flow should be controlled carefully so that the air convection does not break the fiber during the drawing process. During the tapering process, we monitored both the inter-modal interference and the insertion loss of the fiber using the ASE source in conjunction with the OSA. The flame is applied to the fiber in an optimized angle to make sure the heat distribution is homogeneous.

The MLR is fabricated by coiling the microfiber onto itself using two surface attractions, Van der Waals force and electrostatic force, which kept the loop stable since the forces overcame the elastic force to make the fiber straight. The fabricated MLR is laid on an earlier prepared glass plate with a thin and flat layer of low refractive index material to address the temporal stability of the device as shown in Figure 2. A bare MLR may be exposed to dust and moisture that can cause temporal loss instability to the device. The thickness of the low refractive index material is approximately $0.5 \mathrm{~mm}$ which is thick enough to prevent leakage of optical power from the microfiber to the glass plate. Some uncured resin is also applied on surrounding the MLR before it is sandwiched by another glass plate with the same low refractive index resin layer from the top. It is essentially important to ensure that minimum air bubbles and impurity are trapped around the fiber area between the two plates. This is to prevent refractive index non-uniformity in the surrounding of microfiber that may introduce loss to the system. The uncured resin is solidified by the UV light exposure for 3 7 minutes and the optical properties of the MLR are stabilized. The packaged MLR is located in an oven with temperature control to investigate how the comb spectrum changes with the temperature.

\section{RESULT AND DISCUSSION}

A MLR has a comb transmission spectrum similar to that of a Fabry-Perot filter. The resonant wavelength must satisfy $\lambda_{m}=2 \pi R n_{\text {eff }} / m$, where $R$ is the radius of the ring, $n_{\text {eff }}$ is the 


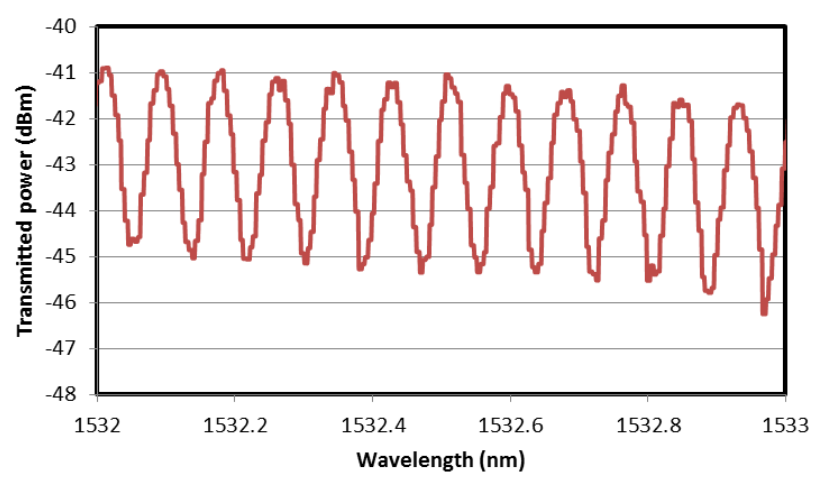

FIG. 3 Transmission spectrum of the fabricated MLR.

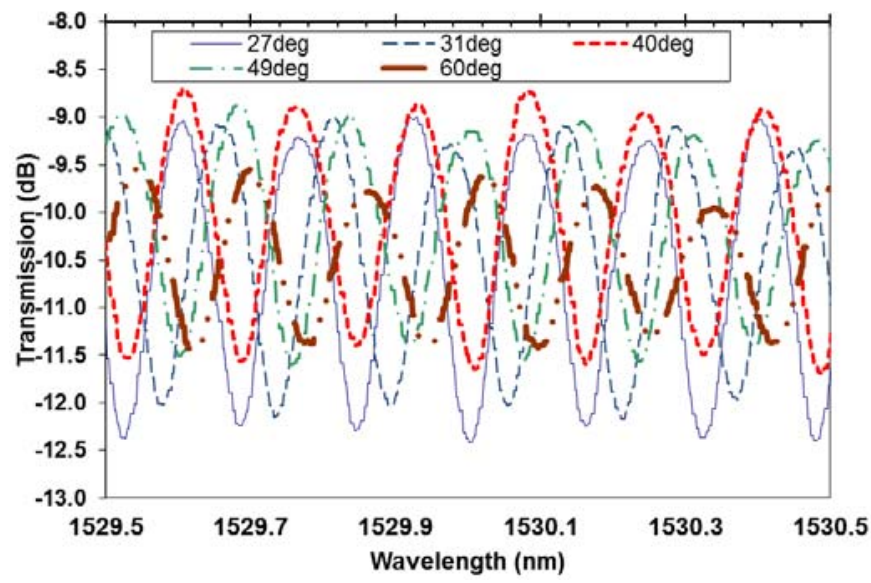

FIG. 4 Transmission spectra of the fabricated MLR obtained at various temperatures.

effective index of the ring, $m$ is the resonant mode number. The resonant frequency spacing, i.e. Free Spectral Range (FSR), is given by [13];

$$
\Delta v_{F S R} \approx \frac{\lambda^{2}}{2 \pi R n_{\text {eff }}}
$$

where $c$ is the velocity of light in vacuum. The quality factor of a ring resonator can be expressed as

$$
Q=\frac{\lambda_{m}}{\Delta \lambda_{F W H M}}
$$

where $\Delta \lambda_{F W H M}$ is the $3 \mathrm{~dB}$ bandwidth of the resonant peak. The lower the coupling coefficient between the ring and straight waveguide, the higher the $Q$ is. If the radius and the coupling coefficient of the ring are chose properly, the desired resonant frequency spacing and bandwidth of the resonant peak can be obtained. The coupling coefficient depends on the overlapping area, effective refractive index and the diameter of tapered fiber. The measured comb transmission spectrum of the MLR is shown in Figure 3, which was obtained by using the ASE source in conjunction with OSA. The resonant response of the MLR is obvious. The extinction ratio is about $4.0 \mathrm{~dB}$, and the FSR is $0.08 \mathrm{~nm}$. The actual radius of MLR is around $3 \mathrm{~mm}$.

The temperature response of the packaged MLR is then investigated. Figure 4 shows the transmission spectrum of an-

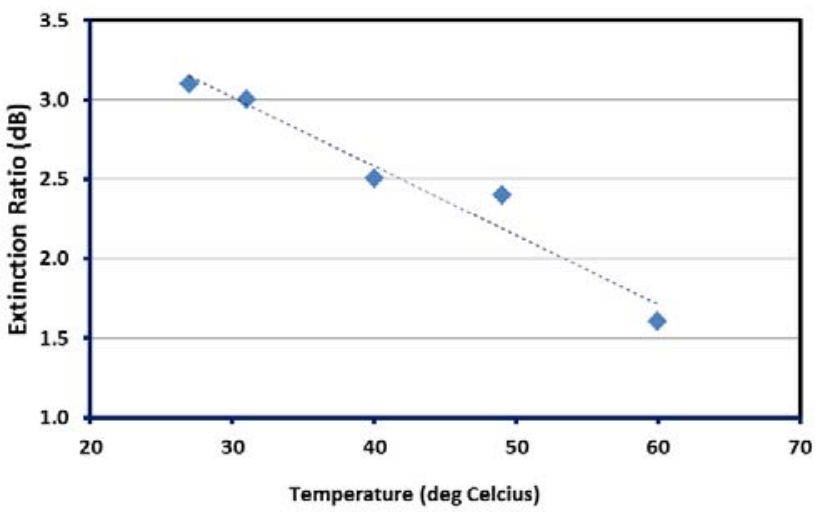

FIG. 5 Extinction ratio of the MLR as a function of the temperature.

other packaged MLR at various temperatures. As shown in this figure, the spacing of the transmission comb spectrum is unchanged with the temperature. However, we observed a linear dependence of the extinction ratio of the MLR on temperature; higher temperature MLRs had a smaller extinction ratio. Figure 5 shows an extinction ratio against the temperature. As seen, the slope of the extinction ratio reduction against temperature was about $0.043 \mathrm{~dB} /{ }^{\circ} \mathrm{C}$. The dependence of the extinction ratio on temperature is due to the change in the material's refractive index, which increase the loss at higher temperature and thus reduces the coupling coefficient of the loop. This affects the quality of the resonator, which in turn reduces the extinction ratio. In the proposed sensor, the optical path length is not much changed with the temperature and therefore, the comb spacing is unchanged as shown in Figure 4.

\section{CONCLUSION}

A MLR has been fabricated and packaged to investigate the temperature response on the transmission spectrum of the device for sensor application. A flame heating method has been used to fabricate microfiber, which is then coiled to form a loop resonator device. The device is embedded into low refractive index polymer for robustness. The spacing of the transmission comb spectrum of the MLR is observed to be unchanged with the temperature. However, the extinction ratio of the spectrum is observed to be linearly decreased with the temperature. The slope of the extinction ratio reduction against temperature was about $0.043 \mathrm{~dB} /{ }^{\circ} \mathrm{C}$. The dependence of the extinction ratio on temperature is due to the change in the material's refractive index.

\section{References}

[1] K. Amarnath, R. Grover, S. Kanakaraju, P. T. Ho, "Electrically pumped InCaAsP-InP microring optical amplifiers and lasers with surface passivation" IEEE Photon. Technol. Lett. 17, 2280-2282 (2005).

[2] S. W. Harun, K. S. Lim, A. A. Jasim, and H. Ahmad, "Dual wavelength erbium-doped fiber laser using a tapered fiber" J. Mod. 
Optic 57, 1362-3044 (2010).

[3] X. Jiang, L. Tong, G. Vienne, X. Guo, A. Tsau, Q. Yang, and D. Yang, "Demonstration of optical microfiber knot resonators" Appl. Phys. Lett. 88 (22), 223501 (2006)

[4] L. Tong, L. Hu, J. Zhang, J. Qiu, Q. Yang, J. Lou, Y. Shen, J. He, and Z. Ye, "Photonic nanowires directly drawn from bulk glasses" Opt. Express 14 (1), 82-87 (2006).

[5] Y. Li, and L. Tong, "Mach-Zehnder interferometers assembled with optical microfibers or nanofibers" Opt. Lett. 33 (4), 303-205 (2008).

[6] X. Jiang, Q. Yang, G. Vienne, Y. Li, L. Tong, J. Zhang, and L. Hu, "Demonstration of microfiber knot laser" Appl. Phys. Lett. 89 (14), 143513 (2006).

[7] H. Ahmad, W. Y. Chong, K. Thambiratnam, M. Z. Zulkifli, P. Poopalan, M. M. M. Thant, and S. W. Harun, "High sensitivity fiber Bragg grating pressure sensor using thin metal diaphragm" IEEE Sens. J. 9 (12), 1654-1659 (2009).

[8] Y. Lou, L. M. Tong, and Z. Z. Ye, "Modeling of silica nanowires for optical sensing" Opt. Express 13, 2135-2140 (2005).
[9] F. Xu, V. Pruneri, V. Finazzi, and G. Brambilla, "High sensitivity refractometric sensor based on embedded optical microfiber loop resonator" in Proceedings of the Conference on Lasers and Electro -Optics/Quantum Electronics and Laser Science Conference and Photonic Applications Systems Technologies, CMJ7 (OSA, San Jose, 2008).

[10] H. C. Nguyen, B. T. Kuhlmey, E. C. Magi, M. J. Steel, P. Domachuk, C. L. Smith, and B. J. Eggleton, "Tapered photonic crystal fibers: Properties, characterization and applications" Appl. Phys. B 81, 377-387 (2005)

[11] V. P. Minkovich, J. Villatoro, D. Monzón-Hernández, S. Calixto, A. B. Sotsky, and L. I. Sotskaya, "Holey fiber tapers with resonance transmission for high-resolution refractive index sensing" 0 pt. Express 13, 7609-7614, (2005).

[12] J. Villatoro, V. P. Minkovich, and D. Monzón-Hernández, "Temperature independent strain sensor made from tapered holey optical fiber" Opt. Lett. 31, 305-307, (2006).

[13] M. Sumetsky, Y. Dulashko, J. M. Fini, A. Hale, and D. J. DiGiovanni, "The microfiber loop resonator: Theory, experiment, and application" J. Lightwave Technol. 24, 242-250 (2006). 